\title{
Die Bedeutung von Partizipation und Scaffolding für die Leistung im naturwissenschaftlichen Sachunterricht in Klassen mit hohem und niedrigem Anteil mehrsprachiger Schüler*innen
}

\author{
Annika Herrmann (D) - Anika Bürgermeister • Kim Lange-Schubert • \\ Henrik Saalbach
}

Eingegangen: 29. Januar 2021 / Angenommen: 14. April 2021 / Online publiziert: 9. Juli 2021

(C) Der/die Autor(en) 2021

Zusammenfassung Studienergebnisse zeigen, dass die Interaktion zwischen Lehrpersonen und Schüler*innen die Lernleistung beeinflusst. Sowohl die aktive Partizipation von Schüler*innen am Unterrichtsgespräch als auch der Einsatz von Unterstützungsstrategien im Sinne des Scaffolding werden dabei als förderlich betrachtet. Es fehlt jedoch an empirischer Forschung insbesondere im Grundschulkontext, die beide Aspekte nicht nur gleichzeitig, sondern auch in ihrer Wechselwirkung betrachtet. Die vorliegende Studie untersucht die Effekte von Partizipation, Scaffolding und ihrer Interaktion auf die Leistung von 1216 Schüler*innen in 54 Grundschulklassen im Fach Sachunterricht. Dabei werden Klassen mit hohem und geringem Anteil mehrsprachiger Schüler*innen vergleichend betrachtet.

Schlüsselwörter Scaffolding · Interaktion · Partizipation · Unterrichtsgespräch · Sachunterricht · Grundschule

\footnotetext{
Annika Herrmann $(\varangle) \cdot$ Dr. Anika Bürgermeister · Prof. Dr. Kim Lange-Schubert ·

Prof. Dr. Henrik Saalbach

Erziehungswissenschaftliche Fakultät, Universität Leipzig, Marschnerstaße 31, 04109 Leipzig,

Deutschland

E-Mail: annika.herrmann@uni-leipzig.de

Dr. Anika Bürgermeister

E-Mail: anika.buergermeister@uni-leipzig.de

Prof. Dr. Kim Lange-Schubert

E-Mail: kim-lange-schubert@uni-leipzig.de

Prof. Dr. Henrik Saalbach

E-Mail: henrik.saalbach@uni-leipzig.de
} 


\title{
The role of participation and scaffolding for science learning in primary school classes with high and low proportion of multilingual pupils
}

\begin{abstract}
Study results show that the interaction between teachers and learners has an effect on performance. Both the active participation of pupils in classroom discourse and teachers' use of supportive strategies in the sense of scaffolding are considered to be beneficial. However, there is a lack of empirical research, especially in the primary school context, which considers both aspects not only simultaneously but also in their interaction. The present study examines the effects of participation, scaffolding and their interaction on science learning with 1216 pupils in 54 primary school classes. Classes with a high and low proportion of multilingual pupils are analysed.
\end{abstract}

Keywords Scaffolding - Interaction - Student participation - Classroom discourse · Science education $\cdot$ Primary school

\section{Einleitung}

Das Unterrichtsgespräch und direkte Instruktion gehören zu den am häufigsten genutzten Methoden bei der Unterrichtsgestaltung (vgl. bspw. Denn et al. 2019). Es ist demnach nicht verwunderlich, dass sich die Unterrichtsforschung in den letzten Jahren vermehrt der Untersuchung der verbalen Interaktionsprozesse im Lehrund Lern-Kontext widmet. Dabei stehen insbesondere die Qualität der Interaktionen und ihre Auswirkung auf die Lernentwicklung im Fokus. Im Sinne eines kokonstruktivistischen Lernverständnisses wird der aktiven Partizipation von Schüler*innen (SuS) dabei ein positiver Effekt auf die Lernleistung zugeschrieben (vgl. bspw. Sedova et al. 2019). Auch der Einsatz von Unterstützungsstrategien durch Lehrpersonen, wie die des Scaffolding, kann sich positiv auf die Lernentwicklung der SuS auswirken (vgl. bspw. Studhalter et al. 2021). Die Effekte von Partizipation und Scaffolding auf die Leistung von SuS wurden bisher größtenteils isoliert betrachtet und nur selten in Bezug zueinander gesetzt. Zudem beziehen sich die meisten Studien auf den Sekundarbereich (bspw. Seidel et al. 2003; Sedova et al. 2019) und einige wenige auf den Elementarbereich (Bürgermeister et al. 2018; Studhalter et al. 2021). Im Primarbereich fehlt es an solchen Untersuchungen (siehe aber Ackermann 2011; Ing et al. 2015).

Die in diesem Artikel vorgestellte Studie untersucht daher den Effekt des Einsatzes von Scaffolding durch die Lehrperson sowie den der Partizipation von SuS auf deren Leistung im naturwissenschaftlichen Sachunterricht der Grundschule. Da Interaktionsprozesse auch durch individuelle Merkmale der SuS sowie Kompositionseffekte der Klasse, wie bspw. Mehrsprachigkeit, beeinflusst werden, werden Klassen mit geringem und hohem Anteil mehrsprachiger SuS vergleichend betrachtet. 


\section{Partizipation und Scaffolding im Unterricht}

Interaktionsprozesse im Unterricht spielen für den Lernerfolg von SuS eine wichtige Rolle (vgl. Ing et al. 2015; Sedova et al. 2019). Diese Erkenntnis spiegelt sich im kokonstruktivistischen Lernverständnis als einem Grundsatz der Didaktik der Grundschule wider. In der Sachunterrichtsdidaktik zeigt sich dieses Lernverständnis in den Conceptual-Change-Theorien, den Ansätzen zur individuellen Förderung (vgl. Hartinger et al. 2014) sowie dem genetisch orientierten Sachunterricht (vgl. Möller 2007). Entsprechend eines ko-konstruktivistischen Lernverständnisses können Kinder nur im Austausch mit ihrer Umwelt bedeutende kognitive Funktionen ausbilden (vgl. Wygotski 1987). Die Interaktion wird demnach als eine Grundlage für den Lernprozess angesehen. In diesem Zusammenhang wurde der Begriff der ,Zone der nächsten Entwicklung“ geprägt, die als ,[d]as Gebiet der noch nicht ausgereiften, jedoch reifenden Prozesse“ beschrieben wird (Wygotski 1987, S. 83). Damit werden Aufgaben oder Problemstellungen beschrieben, die das Kind noch nicht allein, jedoch mit Unterstützung eines fähigen Partners bewältigen kann.

Für diese Ko-Konstruktion von Wissen ist die aktive Partizipation von SuS notwendig (vgl. Wygotski 1987; Mercer und Dawes 2014). Diese Partizipation äußert sich durch eine aktive Beteiligung der SuS an Gesprächen und Diskussionen im Unterricht, die es ihnen ermöglicht, Bedeutungen und Wissen in einem Interaktionsprozess inhaltsbezogen zu konstruieren (vgl. Fthenakis 2009). Durch aktive verbale Partizipation können SuS neues Wissen aufbauen und vertiefen sowie vorhandene Konzepte umstrukturieren (vgl. Decristan et al. 2020). Selbst stille SuS profitieren von einem Klassenumfeld, in dem sich andere SuS aktiv verbal beteiligen (vgl. Inagaki et al. 1998; Pauli und Lipowsky 2007).

Obwohl die aktive verbale Partizipation einen didaktischen Grundsatz darstellt, zeigen Studien, dass Lehrpersonen Unterrichtsgespräche stark dominieren und ihre Redebeiträge deutlich umfangreicher als die der SuS sind (vgl. bspw. Seidel et al. 2003; Bürgermeister et al. 2018; Sedova et al. 2019). Als Begründung für die geringe verbale Beteiligung der SuS wird bspw. die Engführung des Unterrichtsgesprächs durch die Lehrperson genannt, in dem die SuS lediglich als Stichwort- bzw. Antwortgeber gesehen werden (vgl. Seidel et al. 2003). Diese Art der Unterrichtsgestaltung erschwert ein ko-konstruktives Lernen.

Über den Effekt von Partizipation gibt es widersprüchliche Studienergebnisse. Zudem liegen für die Grundschule und insbesondere für den Sachunterricht kaum Befunde vor. Ackermann (2011) kommt bei ihrer Untersuchung zu dem Ergebnis, dass die Häufigkeit von Redebeiträgen der SuS in einigen Klassen im Mathematikunterricht in der Grundschule einen positiven Effekt auf deren Leistung hat. Bei einer der vier untersuchten Klassen zeigte sich jedoch ein negativer Zusammenhang. In einer Studie von Pauli und Lipowsky (2007) hatte die Anzahl an Redebeiträgen der SuS zwar keinen Effekt auf ihre Leistung, sie beeinflusste aber das kognitive und motivationale-emotionale Selbsterleben. Die unterschiedlichen Ergebnisse zeigen, dass weiterhin Forschungsbedarf besteht.

Eine Möglichkeit für die Unterstützung von SuS durch die Lehrpersonen mit Blick auf die Zone der nächsten Entwicklung sind Scaffolding-Strategien. Dabei stellt die Lehrperson den SuS ein Lerngerüst (,scaffold“) bereit, durch das der Lernprozess 
strukturiert wird, die SuS zum weiteren Denken angeregt und zur Bewältigung einer Aufgabe befähigt werden (vgl. Wood et al. 1976). Dieses „Gerüst“ wird bei zunehmenden Lernfortschritten wieder reduziert (vgl. van de Pol et al. 2015). Van de Pol et al. (2015) führen drei wichtige Merkmale des Scaffoldings an: (1) Die Lehrperson muss das Kompetenzniveau der SuS bestimmen und eine daran angepasste Unterstützungsmaßnahme anwenden (contingency); (2) anschließend wird die Unterstützung schrittweise wieder abgebaut (fading); und schließlich (3) wird die Verantwortung ganz auf die SuS übertragen (transfer). Scaffolding umfasst dabei sowohl strukturierende (bspw.: Komplexitätsreduktion, Teilschritte bilden) als auch problematisierende (bspw.: Conceptual Change anregen) Maßnahmen (vgl. Kleickmann et al. 2010; Studhalter et al. 2021). Es vereint damit Aspekte der konstruktiven Unterstützung und der kognitiven Aktivierung, wie sie im Kontext der Unterrichtsqualitätsforschung definiert werden (vgl. Kleickmann et al. 2010). Insbesondere im Sachunterricht kann verbales Scaffolding zu einer konzeptuellen Restrukturierung im Sinne des Conceptual Change anregen (vgl. Leuchter und Saalbach 2014). Empirische Befunde zeigen positive Effekte von verbalem Scaffolding auf den Lernerfolg (vgl. Mannel et al. 2019; Studhalter et al. 2021).

Es wird deutlich, dass sowohl aktive verbale Partizipation als auch Scaffolding lernförderlich wirken können. Die verbale Partizipation des Kindes ist dabei eine notwendige Voraussetzung für die passgenaue Auswahl der Scaffolding-Strategie. Denn aktive Partizipation ermöglicht der jeweiligen Lehrperson Informationen über den Wissensstand der Kinder zu gewinnen und diese für eine gezielte Lernunterstützung in der Zone der nächsten Entwicklung zu nutzen: Das Kind verbalisiert seine Ideen und Konzepte, die Lehrperson kann daraufhin das Kompetenzniveau diagnostizieren und den weiteren Unterricht und ihre Unterstützung adaptiv gestalten (vgl. Bürgermeister und Saalbach 2018; Wygotski 1987). Die Partizipation der SuS kann wiederum durch Scaffolding gefördert werden (vgl. Muhonen et al. 2016). Durch Scaffolding-Strategien werden SuS zu verbalen Äußerungen motiviert, die Einblick in den Wissensstand und in die Konzepte der Kinder geben. Diese Wechselwirkung wurde bisher kaum empirisch untersucht. Allein für den Elementarbereich liegen Befunde vor (Bürgermeister et al. 2018). Demnach hatte aktive Partizipation besonders dann einen positiven Effekt auf die Leistung, wenn die Lehrperson die ScaffoldingStrategie „Aktivierung von Vorwissen“ einsetzte. Im Hinblick auf den naturwissenschaftlichen Unterricht in der Grundschule fehlt es jedoch bisher an entsprechenden Studien.

\section{Die Bedeutung von Mehrsprachigkeit}

Die Lernleistung wird neben der Partizipation und dem Einsatz von Unterstützungsmaßnahmen auch maßgeblich durch individuelle Merkmale der SuS sowie Merkmale der Klassenzusammensetzung, sogenannte Kompositionsmerkmale, beeinflusst (vgl. Biedermann et al. 2015; Kähler et al. 2021). In der hier vorliegenden Studie wird das Kompositionsmerkmal der Mehrsprachigkeit in den Fokus gestellt. Die Gruppe mehrsprachiger SuS in Deutschland ist sehr heterogen (vgl. Martschinke und Kammermeyer 2003; Herwartz-Emden und Küffner 2006; Stanat 2008). Die von 
uns verwendete Bezeichnung „mehrsprachige SuS“ bezieht sich im Folgenden auf Kinder, die im Alltag mehr als eine Sprache verwenden. Dies schließt auch jene Kinder mit ein, die Deutsch als Zweitsprache erlernt haben. Im Folgenden werden zuerst Befunde zu Effekten von Mehrsprachigkeit auf Individualebene vorgestellt und anschließend Kompositionseffekte und das Anliegen der Studie erläutert.

Sprache ist ein bedeutender Faktor für das fachliche Lernen und wird als „Konstruktionsmittel für fachliche Verstehensprozesse" (Leisen 2015, S. 135) bezeichnet. Im vorschulischen naturwissenschaftlichen Lernen und in der Sachunterrichtsdidaktik wird die Bedeutung eines sprachsensiblen Unterrichts betont (vgl. Hardy et al. 2019; Giest et al. 2021). Das zentrale Ziel des Sachunterrichts ist die Umwelterschließung und die Befähigung zum Handeln in der Welt (vgl. Kahlert 2009). Der Sachunterricht verbindet hierbei fachliches und sprachliches Lernen (vgl. Quehl und Trapp 2013). Deshalb ist es insbesondere in diesem Fach wichtig zu untersuchen, wie SuS mit unterschiedlichen sprachlichen Ausgangssituationen gefördert werden können, da im Sinne einer durchgängigen Sprachbildung allen Kindern die Möglichkeit zur Partizipation im Unterricht ermöglicht und sprachliche Hürden abgebaut werden sollten (vgl. Quehl und Trapp 2013). Studienergebnisse zeigen, dass Kinder mit geringeren sprachlichen und kognitiven Fähigkeiten sowie SuS mit Migrationshintergrund weniger häufig in Unterrichtsgespräche einbezogen werden als ihre Klassenkamerad*innen (vgl. bspw. Decristan et al. 2020). Von dieser Benachteiligung sind oftmals mehrsprachige SuS betroffen. Studienbefunde deuten darauf hin, dass Mehrsprachigkeit häufig mit einer schwächeren Sprachkompetenz in der Unterrichtssprache einhergeht (vgl. Hartig et al. 2008; Dubowy et al. 2008). In den internationalen Vergleichsstudien zeigt sich des Weiteren, dass SuS, deren Erstsprache nicht Deutsch ist, geringere Ergebnisse als ihre Mitschüler*innen erreichen (vgl. Reiss et al. 2016; Bos et al. 2017; Kempert et al. 2019). In diesen Studien zeigt sich außerdem ein besonders starker Zusammenhang von sprachlichen Fähigkeiten und naturwissenschaftlichen Kompetenzen. Während sich für die Fächer Deutsch und Mathematik über die Jahre ein Rückgang der Disparitäten erkennen lässt, bleiben sie in den Naturwissenschaften gleichbleibend hoch (vgl. Reiss et al. 2016). Die sprachlich bedingten Leistungsdisparitäten können sowohl auf die kommunikative Funktion als auch auf die kognitive Funktion von Sprache im fachlichen Lernen zurückgeführt werden (Kempert et al. 2019). Im Hinblick auf die kommunikative Funktion können schwache sprachliche Fähigkeiten Probleme im Verständnis der Erklärungen oder der Testaufgaben hervorrufen. So sind etwa die in Vergleichsstudien eingesetzten kompetenzorientierten Aufgaben häufig sprachlich stark kontextualisiert und damit in ihrer Schwierigkeit möglicherweise sprachkompetenzabhängig (Haag et al. 2013). Die kognitive Funktion beschreibt Sprache als zentrales Medium der Wissenskonstruktion. Danach führen begrenzte sprachliche Fähigkeiten zu Problemen im Verständnis zentraler Konzepte und bei prozessbezogenen Kompetenzen, wie etwa dem Argumentieren oder Problemlösen (vgl. Saalbach et al. 2016). Die sprachlich bedingten Leistungsunterschiede entstehen nicht erst in der Sekundarstufe, sondern sind bereits im Elementarbereich zu erkennen (vgl. Bürgermeister et al. 2018) und bleiben bestehen (vgl. Reiss et al. 2016).

Nicht nur individuelle Voraussetzungen von SuS zeigen Effekte auf die Leistung, sondern auch die Klassenkomposition. In einer Studie von Kähler et al. (2021) wurde 
die Entwicklung der naturwissenschaftlichen Kompetenz von SuS an Grundschulen unter Berücksichtigung von individuellen und Klassenmerkmalen untersucht. Es stellte sich heraus, dass Klassenmerkmale zur Varianzaufklärung beitrugen. Unter anderem erwies sich der Effekt des SES (sozioökonomischer Status) als signifikant. SuS in Klassen mit einem hohen Anteil von Kindern mit geringem SES zeigten einen geringeren Kompetenzzuwachs als $\mathrm{SuS}$ in Klassen mit einem geringen Anteil von Kindern mit geringem SES (vgl. ebd.). In dieser Studie wurde auch der Migrationshintergrund auf Klassenebene untersucht. Hier zeigte sich kein eigenständiger, jedoch ein indirekter Effekt im Zusammenhang mit der Vorleistung auf den Zuwachs der naturwissenschaftlichen Kompetenz (vgl. ebd.). Im nationalen Bildungsbericht Österreichs 2015 wurden ebenfalls Kompositionseffekte berichtet. Hier zeigten sich signifikante Effekte des Sozialstatus und der nicht-deutschen Familiensprache auf die mathematische Leistung in vierten und achten Klassen, wobei die Effektstärken in der Sekundarstufe stärker waren (vgl. Biedermann et al. 2015). Das Kompositionsmerkmal Mehrsprachigkeit wurde bisher noch nicht eigenständig untersucht. Außerdem wurden Kompositionseffekte weitestgehend in Bezug auf Leistung untersucht, Befunde zu ihrer Auswirkung auf die Partizipation fehlen bislang. Ausgehend von den vorgestellten Ergebnissen zu individuellen Effekten von Mehrsprachigkeit auf Partizipation gilt es zu untersuchen, ob sich auch auf Klassenebene ein negativer Effekt zeigt, die SuS in Klassen mit einem hohen Anteil mehrsprachiger SuS also weniger verbale Partizipation zeigen als in Klassen mit einem geringen Anteil mehrsprachiger SuS. Die vorliegende Studie setzt hier an und untersucht Mehrsprachigkeit als Kompositionsmerkmal tiefergehender.

\section{Fragestellungen und Hypothesen}

Ziel der Studie ist es, den Effekt von Partizipation der SuS, Scaffolding durch die Lehrpersonen sowie der Interaktion von Partizipation und Scaffolding auf die Lernleistung im Sachunterricht $\mathrm{zu}$ untersuchen und dabei Klassen mit geringem $(<25 \%)$ und hohem $(\geq 25 \%)$ Anteil mehrsprachiger SuS vergleichend zu betrachten.

Es ergeben sich die folgenden Fragestellungen:

Fragestellung 1 Wie unterscheidet sich die Partizipation von SuS am Diskurs im naturwissenschaftlichen Unterricht in Klassen mit geringem und hohem Anteil mehrsprachiger SuS?

Hypothese 1 Ausgehend von empirischen Befunden zur individuellen Partizipation von SuS wird angenommen, dass die Partizipation der SuS in Klassen mit hohem Anteil mehrsprachiger SuS geringer als in Klassen mit geringem Anteil mehrsprachiger SuS ist und sich die Effekte somit auf Klassenebene reproduzieren lassen.

Fragestellung 2 Gibt es einen Effekt der Partizipation der SuS auf die Leistung in Klassen mit geringem und hohem Anteil mehrsprachiger SuS? 
Hypothese 2 Es wird vermutet, dass die Partizipation einen positiven Effekt auf die Leistung hat und somit eine höhere Anzahl an gesprochenen Worten in der Klasse zu einem besseren Ergebnis im Post-Test beiträgt.

Fragestellung 3 Gibt es einen Effekt des Scaffolding der Lehrperson auf die Leistung in Klassen mit geringem und hohem Anteil mehrsprachiger SuS?

Hypothese 3 Es wird angenommen, dass Scaffolding durch die Lehrperson einen positiven Effekt auf die Leistung der SuS hat und somit ein vermehrter Einsatz von Scaffolding-Maßnahmen während des Unterrichts zu einer besseren Leistung im Post-Test beiträgt.

Fragestellung 4 Gibt es einen Effekt der Interaktion von Partizipation und Scaffolding auf die Leistung in Klassen mit geringem und hohem Anteil mehrsprachiger SuS?

Hypothese 4 Ausgehend von Befunden aus dem Elementarbereich (vgl. Bürgermeister et al. 2018) wird vermutet, dass es einen positiven Interaktionseffekt von Partizipation und Scaffolding auf die Leistung gibt.

\section{Methode}

Um die vorgestellten Forschungsfragen zu beantworten, wurde eine Re-Analyse der Studie „PLUS“ (u. a. Ewerhardy et al. 2012; Tröbst et al. 2016) durchgeführt. An dieser Studie nahmen 1326 SuS aus 60 vierten Klassen an Grundschulen und 1354 SuS aus 54 sechsten Klassen der Sekundarstufe teil.

\subsection{Stichprobe}

Der Fokus für die Re-Analyse liegt auf den Grundschulklassen, wobei sechs Klassen ausgeschlossen wurden, da von ihnen entweder kein Video und somit kein Transkript vorlag oder aufgrund fehlender Elternfragebögen die Informationen zur Mehrsprachigkeit der Kinder nicht vorlagen. Die Stichprobe besteht somit aus 54 vierten Klassen mit 1216 SuS. 46,3\% der teilnehmenden Kinder sind weiblich und ihr Durchschnittsalter beträgt 10,25 Jahre $(S D=0,62) .45$ der 54 Lehrpersonen sind weiblich und ihre durchschnittliche Lehrerfahrung beträgt 14,47 Jahre $(S D=11,48)$.

\subsection{Design}

Die teilnehmenden Lehrpersonen führten eine Unterrichtseinheit, bestehend aus drei 90-minütigen Unterrichtsstunden, zum Thema „Aggregatzustände und ihre Übergänge am Beispiel Wasser" durch. Die erste 90-minütige Unterrichtssequenz wurde dabei videografiert. Die angefertigten Unterrichtsvideos und die daraus entstandenen Transkripte bilden die Grundlage für die Re-Analyse. Die Lehrpersonen bekamen die Instruktion, während des Unterrichts auf Wasser in seiner flüssigen und 
Tab. 1 Schematischer Ablauf Studie „PLUS“

\begin{tabular}{|c|c|c|}
\hline Vor dem Unterricht & Während des Unterrichts & Nach dem Unterricht \\
\hline SuS-Leistungstest & Drei 90-minütige Unterrichtsstunden & SuS-Leistungstest \\
\hline SuS-Fragebogen & Videographie der ersten & SuS-Fragebogen \\
\hline Elternfragebogen & Unterrichtsdoppelstunde & \\
\hline$\square-$ & & \\
\hline
\end{tabular}

gasförmigen Form einzugehen und dabei Faktoren, die zur Beschleunigung bzw. Verlangsamung des Verdampfens führen, zu thematisieren (vgl. Tröbst et al. 2016). Durch die Vorgaben wurde die Unterrichtssituation teilweise standardisiert und die einzelnen Klassen sind mit einander vergleichbar. In der Studie wurden außerdem Leistungstests und Fragebögen eingesetzt, die im Abschnitt „Instrumente“ genauer beschrieben werden. Der Ablauf der Studie ist in der Tab. 1 skizziert.
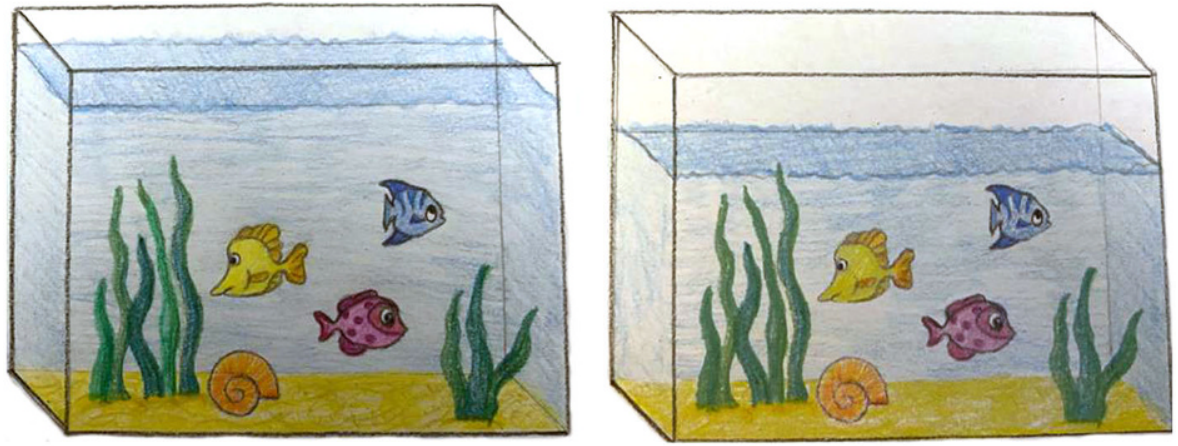

Aus dem Aquarium verschwindet mit der Zeit immer mehr Wasser.

Wie nennt man diesen Vorgang?

囚 Kreuze nach jedem Wort richtig oder falsch an!

\begin{tabular}{|l|l|l|}
\hline & Richtig & Falsch \\
\hline Schmelzen & & \\
\hline Sieden & & \\
\hline Kondensieren & & \\
\hline Verdunsten & & \\
\hline
\end{tabular}

Abb. 1 Multiple-Choice-Frage zum Begriffswissen 


\subsection{Instrumente}

\subsubsection{Test zur Erfassung der Leistung der SuS}

Zur Erfassung der Leistung wurde ein Prä-Post-Test-Verfahren eingesetzt. Vor der ersten Unterrichtseinheit nahmen die SuS am Prä-Test zum Thema ,Aggregatzustände“" teil. Der Post-Test wurde am Ende der Unterrichtseinheit mit den Kindern durchgeführt. Der Score im Prä-Test stellt das Vorwissen der SuS als individuelles Merkmal dar. Der Test wurde im Rahmen des PLUS-Projekts entwickelt und erprobt. Die Tests wurden im Paper-Pencil-Format durchgeführt und bestanden aus 24 Multiple-Choice-Fragen bzw. Multiple-Select-Fragen (vgl. Lange et al. 2015). 15 Aufgaben lassen sich dabei dem Begriffswissen zuordnen, bei dem die Kinder
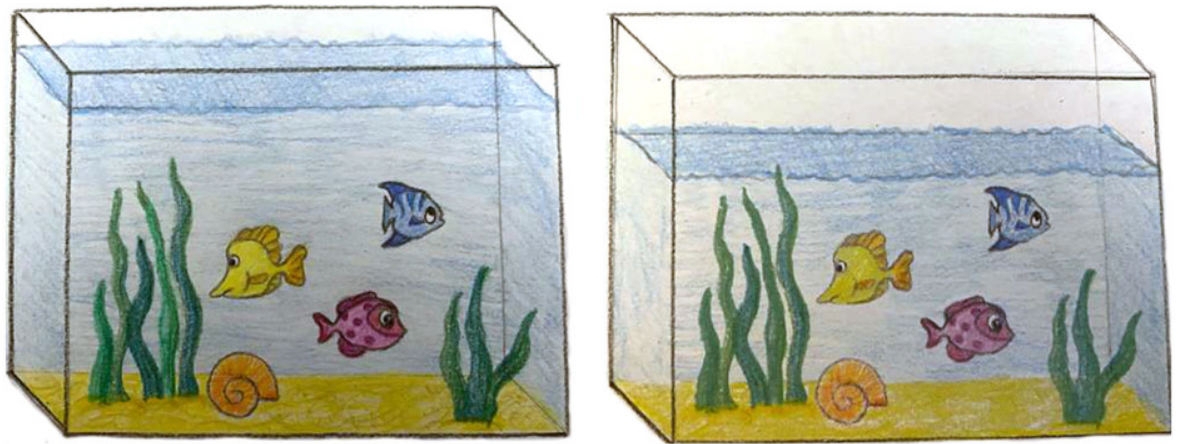

Anna hat ein neues Aquarium. Sie bemerkt, dass nach ein paar Tagen weniger Wasser im Aquarium ist. Anna untersucht das ganze Aquarium, findet aber keine Löcher oder Risse.

Was ist mit dem Wasser aus dem Aquarium passiert?

囚 Kreuze nach jeder Erklärung richtig oder falsch an!

\begin{tabular}{|l|l|l|}
\hline & Richtig & Falsch \\
\hline $\begin{array}{l}\text { Das Wasser ist im Sand versickert und ist in dem Boden } \\
\text { verteilt. }\end{array}$ & & \\
\hline $\begin{array}{l}\text { Das Wasser hat sich in nicht sichtbares Wasser verwandelt } \\
\text { und ist in der Luft verteilt. }\end{array}$ & & \\
\hline $\begin{array}{l}\text { Das Wasser ist von den Sonnenstrahlen in den Himmel } \\
\text { gezogen worden. }\end{array}$ & $\begin{array}{l}\text { Das Wasser hat sich in nicht sichtbaren Sauerstoff verwandelt } \\
\text { und ist in der Luft verteilt. }\end{array}$ & \\
\hline Das Wasser ist verschwunden und ist nirgendwo. & \\
\hline
\end{tabular}

Abb. 2 Multiple-Choice-Frage zum konzeptuellen Wissen 
passende Fachbegriffe auswählen sollen (vgl. ebd; siehe Abb. 1). Die restlichen 9 Aufgaben erfragen das konzeptuelle Wissen der SuS, indem passende Erklärungen für Verdunstungs- und Kondensationsvorgänge ausgewählt werden sollen (vgl. ebd; siehe Abb. 2). Cronbachs Alpha betrug für den Prä-Test 0,67 und für den PostTest 0,79 (vgl. ebd).

\subsubsection{Elternfragebogen}

Im Elternfragebogen wurden demografische Merkmale der SuS erfasst. Die Eltern machten u. a. Angaben zum sprachlichen Hintergrund des Kindes. Auf dieser Grundlage wurde im Rahmen der Re-Analyse bestimmt, ob ein Kind ein- oder mehrsprachig ist. Ein Kind wird als mehrsprachig eingestuft, wenn mindestens eines der folgenden Merkmale erfüllt wird (vgl. Herzog-Punzenberger und Schnell 2012):

1. Das Kind hat mindestens eine andere Sprache als Deutsch zuerst erworben.

2. In der Familie des Kindes wird mindestens eine andere Sprache als Deutsch vorrangig gesprochen.

3. Im Haushalt wird mindestens eine andere Sprache als Deutsch vorrangig gesprochen.

\subsubsection{Klassen mit hohem und geringem Anteil mehrsprachiger SuS}

Um Klassen mit geringem und hohem Anteil mehrsprachiger SuS vergleichend betrachten zu können, wurde der relative Anteil mehrsprachiger SuS pro Klasse ermittelt. Liegt der Anteil mehrsprachiger SuS unter 25\%, wird diese Klasse als eine Klasse mit geringem Anteil mehrsprachiger SuS betrachtet. Liegt der Wert bei mindestens $25 \%$, so wird die Klasse als eine Klasse mit hohem Anteil mehrsprachiger SuS betrachtet. Die Stichprobe besteht nach dieser Einteilung aus 41 Klassen mit geringem und 13 Klassen mit hohem Anteil mehrsprachiger SuS (vgl. Tab. 2). In der hier vorgestellten Studie wurde ein Cut-Off-Wert für den Anteil an mehrsprachigen SuS gesetzt. Die Einteilung in zwei Gruppen wurde gewählt, um die Ergebnisse der Regressionsmodelle direkt miteinander vergleichen zu können.

\subsubsection{Kodiersystem zur Erfassung der Partizipation}

Um die Partizipation der SuS am Unterrichtsgespräch zu messen, wurde ein Kodiersystem von Bürgermeister et al. (2018; vgl. Ackermann 2011) eingesetzt. Krippendorffs Alpha (Hayes und Krippendorff 2007) betrug 0,99. Dabei wurden sowohl die

Tab. 2 Stichprobe

\begin{tabular}{|c|c|c|c|c|c|}
\hline & $\begin{array}{l}N \text { Klas- } \\
\text { sen }\end{array}$ & $\begin{array}{l}N \\
\mathrm{SuS}\end{array}$ & $\begin{array}{l}\text { Anteil mehrspra- } \\
\text { chiger SuS }\end{array}$ & Alter & Geschlecht \\
\hline $\begin{array}{l}\text { Klassen mit geringem Anteil } \\
\text { mehrsprachiger SuS }\end{array}$ & 41 & 939 & $\begin{array}{l}M=11,46 \% \\
S D=6,60\end{array}$ & $\begin{array}{l}M=10,22 \\
S D=0,62\end{array}$ & $45,6 \%$ 우 \\
\hline $\begin{array}{l}\text { Klassen mit hohem Anteil } \\
\text { mehrsprachiger SuS }\end{array}$ & 13 & 277 & $\begin{array}{l}M=39,38 \% \\
S D=11,50\end{array}$ & $\begin{array}{l}M=10,37 \\
S D=0,62\end{array}$ & $46,7 \%$ 우 \\
\hline
\end{tabular}


Anzahl der Redebeiträge als auch die einzelnen gesprochenen Wörter erfasst. Als Redebeitrag werden einzelne Turns bzw. Gesprächsbeiträge der Personen definiert. Ein Redebeitrag wird dabei durch einen Sprecher- oder Adressatenwechsel begrenzt. Für diese Analyse wurden die Transkripte in das Programm „MAXQDA Analytics Pro“ eingelesen. Die so gewonnenen Daten wurden auf Gruppenebene aggregiert und somit für alle SuS einer Klasse gemeinsam erfasst. In den späteren Analysen wurde Partizipation durch die gesprochenen Wörter operationalisiert. Eine höhere Partizipation bedeutet demzufolge eine höhere Anzahl gesprochener Wörter.

\subsubsection{Skalen zur Erfassung der Scaffolding-Strategien}

Zur Auswertung der Scaffolding-Strategien wurden Daten einer vorherigen Studie genutzt (Ewerhardy et al. 2012) Ewerhardy entwickelte ein hoch-inferentes Videoanalyseinstrument zur Beschreibung des konstruktivistisch orientierten naturwissenschaftlichen Sachunterrichts. In der hier vorgestellten Studie wurde als Proxy für die Scaffolding-Variable die Skala „Umgang mit Schülervorstellungen“ genutzt. Die Items dieser Skala sind:

1. das Vorwissen der Lernenden explorieren

2. die aufgebauten Schülervorstellungen unterrichtsbegleitend explorieren

3. das Erkennen von Unzulänglichkeiten in den Vorstellungen der Lernenden herbeiführen

4. die Vorstellungen der Lernenden für den Aufbau angemessener Konzepte nutzen

5. eine Fehlerkultur sicherstellen

6. eine geeignete Auswahl an Versuchen/Materialien vornehmen

7. eine Evidenzbasierung für den Auf- bzw. Umbau von Schülervorstellungen herstellen

Die Items der Skala überschneiden sich mit anerkannten Scaffolding-Maßnahmen, wie bspw. der Vorwissensaktivierung, an Vorstellungen von SuS anknüpfen und Förderung des Conceptual Change, weswegen die Skala in der hier vorgestellten Studie zur Operationalisierung des Scaffolding genutzt wird.

Die Rater stuften den Unterricht auf einer vierstufigen Likert-Skala ein. Ein höherer Wert dieser Einstufung entspricht somit einem höheren Wert der Variable Scaffolding. Die Interrater-Reliabilität ist als gut einzustufen (ICCunjust $=0,82$, Cronbachs Alpha $=0,82$.) Die Variable Scaffolding wurde auf Gruppenebene erfasst. Bei der Erfassung des Scaffolding auf Gruppenebene wird angenommen, dass sich das exemplarische Verhalten der Lehrperson auf den von ihr durchgeführten Unterricht im Allgemeinen und ihren Unterrichtsstil übertragen lässt und somit Aussagen nicht nur für einzelne SuS, sondern für die gesamte Klasse getroffen werden können.

\subsubsection{Kontrollvariablen}

In die Re-Analyse wurden zusätzlich zu den vorgestellten Prädiktorvariablen auch Kontrollvariablen einbezogen. Diese Variablen bilden das Vorwissen, das mithilfe des Leistungstests erfasst wurde, sowie die allgemeinen kognitiven Fähigkeiten der SuS. Die Daten zu den allgemeinen kognitiven Fähigkeiten stammen ebenfalls 
aus der PLUS-Studie, dafür wurden zwei Subskalen des CFT 20-R (Weiß 2005) verwendet. Cronbach's Alpha beträgt 0,65 (vgl. Tröbst et al. 2016).

\subsection{Statistische Analysen}

Zuerst wurde deskriptiv untersucht, wie sich die verbale Beteiligung von SuS und Lehrpersonen in allen Klassen verteilt und T-Tests wurden durchgeführt. Um herauszufinden, ob es Unterschiede in der Partizipation von $\mathrm{SuS}$ in Klassen mit geringem und hohem Anteil mehrsprachiger SuS gibt, wurden zunächst T-Tests auf Klassenebene in Bezug auf die Wortanzahl durchgeführt, die nach der MehrsprachigkeitsVariable getrennt wurden. Anschließend wurden die Effektstärken bestimmt.

Zur Beantwortung der Fragestellungen 2, 3 und 4 wurde eine MultigruppenMehrebenen-Regressionsanalyse mithilfe des Programms „Mplus“ (Muthén und Muthén 2017) durchgeführt. Das Kriterium für diese Analyse stellt die Leistung im Post-Test dar. Die Prädiktoren auf Individualebene sind das Wissen im Prä-Test und die allgemeinen kognitiven Fähigkeiten. Auf Klassenebene wurden die Prädiktoren Partizipation (operationalisiert über die Wortanzahl), Scaffolding und die Interaktion zwischen Partizipation und Scaffolding analysiert. Eine Multigruppenanalyse wurde durchgeführt, da für die abhängige Variable Leistung der ICC bei 0,199 liegt. Das Ergebnis des ICC zeigt, dass 19,9\% der Unterschiede im Leistungstest auf die Klasse, in der sich ein*e Schüler*in befindet, zurückzuführen sind, weshalb es sinnvoll ist, unterschiedliche Gruppen in der Regressionsanalyse zu untersuchen.

\section{Ergebnisse}

\subsection{Deskriptive Statistik}

Der erreichte Score des Post-Tests unterschied sich nicht signifikant zwischen Klassen mit geringem $(M=12,13, S D=4,59)$ und hohem $(M=11,17, S D=4,54)$ Anteil mehrsprachiger SuS $(p=0,46)$.

Bei der Untersuchung der Partizipation über alle Klassen hinweg zeigte sich, dass die Anzahl der Redebeiträge zwischen SuS und Lehrpersonen sehr ähnlich verteilt

Abb. 3 Verteilung der Redebeiträge und Wortanzahl zwischen SuS und Lehrpersonen

- SuS Lehrpersonen

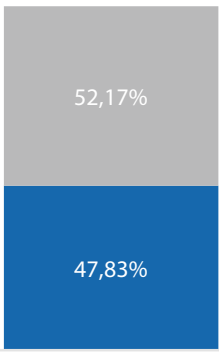

Redebeiträge

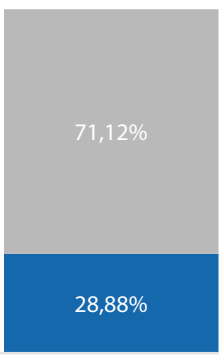

Wortanzahl 
Abb. 4 Partizipation in Klassen mit hohem und geringem Anteil mehrsprachiger SuS
3500

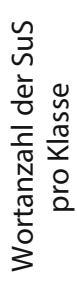

- hoher Anteil mehrsprachiger SuS

- geringer Anteil mehrsprachiger SuS

ist. Durchschnittlich $47,83 \%$ der Redebeiträge lassen sich dabei den Lernenden zuordnen (absolut: $\left.M_{\text {Sus }}=387,78, S D=133,25 ; M_{\text {Lehrpersonen }}=414,19, S D=117,04\right)$. Der Unterschied in der Anzahl der Redebeiträge ist nicht signifikant $(p=0,28)$. Wie in Abb. 3 zu erkennen, ist der Umfang der Redebeiträge, also die Anzahl der gesprochenen Wörter, der Lehrpersonen deutlich höher als der der SuS. So lassen sich durchschnittlich $71,12 \%$ der gesprochenen Wörter den Lehrenden zuordnen (absolut: $M_{\text {SuS }}=2163,12, S D=926,39 ; M_{\text {Lehrpersonen }}=5286,75, S D=1473,97$ ). Dieser Unterschied ist signifikant $(p<0,001)$.

Fragestellung 1 fokussiert sich auf die Partizipation von SuS in Klassen mit geringem und hohem Anteil mehrsprachiger SuS. Die Partizipation wird dabei über die Wortanzahl gemessen. Wie in Abb. 4 zu sehen, zeigt sich, dass die durchschnittliche Wortanzahl in Klassen mit hohem Anteil mehrsprachiger SuS $(M=1812,08$, $S D=872,54)$ niedriger ist als die in Klassen mit geringem Anteil mehrsprachiger SuS $(M=2251,55, S D=969,46)$. Dieser Unterschied ist jedoch nicht signifikant $(p=0,15)$. Bei der Berechnung der Effektstärke zeigt sich ein kleiner Effekt $(d=0,46)$.

Fragestellungen 2,3 und 4 beschäftigen sich mit dem Effekt der Variablen Partizipation, Scaffolding und der Interaktion von Scaffolding und Partizipation auf die Leistung. Dafür wurde eine Multigruppen-Mehrebenen-Regressionsanalyse durchgeführt, deren Ergebnisse in Tab. 3 dargestellt werden.

Auf der Individualebene zeigen sich signifikante Effekte für die Kontrollvariablen Vorwissen und allgemeine kognitive Fähigkeiten in beiden Gruppen. Bei der Betrachtung der Prädiktoren auf Gruppenebene zeigt sich, dass Partizipation in beiden Gruppen keinen signifikanten Effekt auf die Leistung hat. Für die Variable Scaffolding zeigen sich unterschiedliche Ergebnisse für die untersuchten Gruppen. 
Tab. 3 Gesamtmodell der Multigruppen-Mehrebenen-Regressionsanalyse zu Fragestellung 2, 3, 4: Effekt der Prädiktoren auf die Leistung

\begin{tabular}{lll}
\hline Prädiktor & $\begin{array}{l}\text { Leistung } \\
\text { Klassen mit geringem Anteil mehr- } \\
\text { sprachiger SuS }\end{array}$ & $\begin{array}{l}\text { Klassen mit hohem Anteil mehr- } \\
\text { sprachiger SuS }\end{array}$ \\
\hline $\begin{array}{l}\text { Individualebene (E1) } \\
\text { Intercept }\end{array}$ & $0,25^{*}$ & 0,06 \\
Allgemeine kognitive & $\mathbf{0 , 0 8 *}$ & $\mathbf{0 , 1 4 * * *}$ \\
Fähigkeiten & & \\
Vorwissen & $\mathbf{0 , 5 2 * * *}$ & $\mathbf{0 , 5 0 * * *}$ \\
Restvarianz & $0,50^{* * *}$ & $0,50^{* * *}$ \\
$\mathrm{R}^{2}$ & 0,29 & 0,26 \\
Gruppenebene (E2) & & \\
Partizipation & 0,15 & $-0,06$ \\
Scaffolding & 0,04 & $\mathbf{0 , 1 5 *}$ \\
Partizipation $\times$ Scaffolding & $-0,09$ & $\mathbf{0 , 1 4 *}$ \\
Restvarianz & $0,15^{* *}$ & 0,03 \\
$\mathrm{R}^{2}$ & 0,48 & 0,81 \\
\hline
\end{tabular}

Darstellung der standardisierten $\beta$-Koeffizienten $* p<0,05 ; * p<0,01 ; * * *<<0,001$

In Klassen mit geringem Anteil mehrsprachiger SuS konnte kein signifikanter Effekt festgestellt werden. In Klassen mit hohem Anteil mehrsprachiger SuS zeigte sich jedoch ein signifikant positiver Effekt von Scaffolding auf die Leistung. Ein paralleles Ergebnis konnte für den Interaktionseffekt von Partizipation und Scaffolding festgestellt werden. Auch hier ist der Effekt auf die Leistung lediglich in den Klassen mit hohem Anteil mehrsprachiger SuS signifikant.

\section{Diskussion}

Im Hinblick auf die Partizipation der SuS konnten die Ergebnisse aus dem Elementarbereich teilweise reproduziert werden (vgl. Bürgermeister et al. 2018). Während die Anzahl der Redebeiträge der Kinder im Elementarbereich die der Lehrenden überstieg, zeigte sich für den Primarbereich eine nahezu ausgeglichene Verteilung. Erwartungskonform sind die Redebeiträge der Lehrpersonen jedoch deutlich umfangreicher als die der SuS. Obwohl die Verteilung der Redebeiträge zwischen Lehrpersonen und SuS im ersten Moment nicht konform zu den bisherigen Studienbefunden des Schulkontexts zu sein scheint, zeigt die Verteilung der Wortanzahl doch deutlich, dass Lehrpersonen auch im Sachunterricht der Grundschule das Unterrichtsgespräch erheblich dominieren. Das deutet darauf hin, dass Lernende von der Elementar- bis zur Sekundarstufe eher kurze Redebeiträge formulieren und in Interaktionsprozessen als „Stichwortgeber“ zu fungieren scheinen (vgl. Seidel et al. 2003).

Die Ergebnisse zu Fragestellung 1 bestätigen die aufgestellte Hypothese nicht. In den untersuchten Klassen mit hohem Anteil mehrsprachiger SuS ist die Partizipation 
zwar durchschnittlich geringer als in Klassen mit geringem Anteil mehrsprachiger $\mathrm{SuS}$, dieser Unterschied ist jedoch statistisch nicht signifikant. Die Befunde früherer Studien, die zeigen, dass SuS mit geringeren sprachlichen Kompetenzen und/oder Migrationshintergrund weniger häufig in Unterrichtsgespräche einbezogen werden (vgl. bspw. Decristan et al. 2020), konnten wir somit nicht auf Klassenebene reproduzieren.

Ein direkter Effekt von Partizipation auf die Leistung im Sachunterricht konnte weder für SuS in Klassen mit geringem noch mit hohem Anteil mehrsprachiger SuS festgestellt (siehe Tab. 3) und somit die Hypothese zu Fragestellung 2 nicht bestätigt werden. In den Ergebnissen zu Fragestellung 3 und 4 zeigten sich jedoch interessante Effekte. Für Scaffolding ließ sich ein signifikant positiver Effekt auf die Leistung feststellen, jedoch ausschließlich in Klassen mit hohem Anteil mehrsprachiger SuS. Auch der Interaktionseffekt von Scaffolding und Partizipation wirkt nur in Klassen mit hohem Anteil mehrsprachiger SuS signifikant positiv auf die Leistung. Eine mögliche Interpretation dieses Ergebnis ist, dass sich in Klassen mit hohem Anteil mehrsprachiger SuS eine aktive Partizipation der SuS positiv auf die Leistung auswirkt, wenn sie mit Scaffolding-Maßnahmen durch die Lehrperson einhergeht. Das könnte als Hinweis darauf gedeutet werden, dass die Lehrperson durch die aktive verbale Partizipation in der Lage ist, den Wissensstand der SuS zu diagnostizieren und daraufhin passende Scaffolding-Maßnahmen auswählen konnte. Da sich der Interaktionseffekt in den Klassen mit geringem Anteil mehrsprachiger SuS nicht zeigte, darf diese Annahme nur vorsichtig aus den Ergebnissen gebildet werden. Hiermit konnten die aufgestellten Hypothesen zu den Fragestellungen 3 und 4 nur teilweise, in Klassen mit hohem Anteil mehrsprachiger SuS, bestätigt werden. Die Ergebnisse aus dem Elementarbereich, die einen positiven Interaktionseffekt von Scaffolding und Partizipation auf die Leistung zeigen, konnten demnach nur teilweise reproduziert werden (vgl. Bürgermeister et al. 2018). Die Ergebnisse passen jedoch zu Befunden, in denen sich ein Effekt von Unterstützungsstrategien nur für SuS mit geringer Sprachkompetenz in Deutsch zeigte (vgl. Decristan et al. 2015), wobei Mehrsprachigkeit nicht mit geringer Sprachkompetenz gleichgesetzt werden darf. Die hier vorliegenden Ergebnisse stützen also die Annahme, dass SuS in Klassen mit hohem Anteil mehrsprachiger SuS in besonderem Maße von Scaffolding und der Kombination von Scaffolding und aktiver Partizipation profitieren. Dass Scaffolding in der hier vorgestellten Studie nicht per se einen positiven Effekt auf die Leistung zeigte, deutet daraufhin, dass hier kein linearer Zusammenhang angenommen werden darf. Van de Pol et al. (2015) machen in diesem Kontext deutlich, dass Scaffolding immer adaptiv stattfinden muss. Es ist demnach denkbar, dass sich ein Effekt von Scaffolding auf die Leistung zeigt, wenn die Strategien auch hinsichtlich ihrer Adaptivität analysiert werden.

\section{Limitation und Ausblick}

Aufgrund des Studiendesigns als Feldstudie mit relativ großer Stichprobe konnten die Merkmale Partizipation und Scaffolding sowie der Interaktionsterm aus Partizipation und Scaffolding ausschließlich auf Gruppenebene analysiert werden, da 
es (noch) nicht möglich ist, die Äußerungen einzelnen Kindern zuzuordnen. Ein interessanter Ansatzpunkt für weiterführende Studien könnte sein zu erfassen, in welchem Maß sich einzelne SuS am Unterrichtsgespräch beteiligen und wie die Lehrperson mit einzelnen Kindern agiert und welche spezifischen Scaffolding-Strategien sie nutzt, wobei insbesondere die Passung zwischen der Äußerung der SuS und dem Scaffold der Lehrperson in den Fokus genommen werden sollte. Dadurch könnte untersucht werden, wie sich die Merkmale auf die individuelle Leistung des Kindes auswirken.

Für das Merkmal Scaffolding ist weiterführend zu beachten, dass in dieser Studie ein Proxy verwendet wurde. Die Items der verwendeten Skala stimmen zwar mit anerkannten Scaffolding-Strategien überein, jedoch kann mit dieser Skala nicht die Adaptivität des Scaffolding erfasst werden. Laut van de Pol et al. (2015) ist die Adaptivität (contingency) ein Hauptmerkmal für das Gelingen von Scaffolding (vgl. Bürgermeister et al. 2018). Es wäre demnach gewinnbringend, wenn zukünftige Untersuchungen das Scaffolding genauer betrachten und insbesondere analysieren, inwieweit Lehrpersonen die Scaffolding-Strategien adaptiv einsetzen.

In die Multigruppen-Mehrebenen-Regressionsanalyse wurden 41 Klassen mit geringem Anteil mehrsprachiger SuS und 13 Klassen mit hohem Anteil mehrsprachiger SuS einbezogen. Die unterschiedlichen Gruppengrößen stellen eine weitere Limitation der Studie dar. Es wäre lohnenswert bei zukünftigen Studien ähnlich große Gruppen zu bilden und zu untersuchen, ob sich die Effekte von Scaffolding und der Interaktion von Partizipation und Scaffolding reproduzieren lassen. Des Weiteren wurde die Sprachkompetenz der teilnehmenden SuS nicht erhoben und konnte somit nicht in die Analysen einbezogen werden.

\section{Fazit und Implikationen}

In der Grundschuldidaktik wird die Bedeutung von Sprache und Sache sowie die Verbindung von sprachlichem und fachlichem Lernen betont, was sich auch in der Sachunterrichtsdidaktik widerspiegelt (vgl. Leisen 2015; Giest et al. 2021). Die hier vorgestellte Studie zeigt, dass insbesondere SuS in Klassen mit hohem Anteil mehrsprachiger SuS von Scaffolding profitieren. Es scheint also lohnenswert, dass Lehrpersonen die Zusammensetzung einer Lerngruppe bei der Unterrichtsplanung berücksichtigen und speziell in Klassen mit hohem Anteil mehrsprachiger SuS gezielt Scaffolding-Strategien einsetzen, um die SuS sprachlich und fachlich zu fördern und einer Bildungsbenachteiligung entgegenzuwirken. Gleichzeitig sollte auch eine aktive Partizipation der SuS gefördert werden. In der hier vorgestellten Studie zeigte sich die Bedeutung der Interaktion von Partizipation und Scaffolding für die Leistung in Klassen mit hohem Anteil mehrsprachiger SuS. Hiermit wird deutlich, dass Scaffolding besonders effektiv in Verbindung mit einer aktiven Partizipation der SuS ist. Lehrpersonen sollten dementsprechend versuchen, SuS in Gespräche und Diskussionen einzubinden, ihnen Raum bieten, ihre eigenen Vorstellungen und Gedanken zu äußern und somit einen ko-konstruktiven Aufbau von Wissen zu unterstützen. 
Funding Open Access funding enabled and organized by Projekt DEAL.

Open Access Dieser Artikel wird unter der Creative Commons Namensnennung 4.0 International Lizenz veröffentlicht, welche die Nutzung, Vervielfältigung, Bearbeitung, Verbreitung und Wiedergabe in jeglichem Medium und Format erlaubt, sofern Sie den/die ursprünglichen Autor(en) und die Quelle ordnungsgemäß nennen, einen Link zur Creative Commons Lizenz beifügen und angeben, ob Änderungen vorgenommen wurden.

Die in diesem Artikel enthaltenen Bilder und sonstiges Drittmaterial unterliegen ebenfalls der genannten Creative Commons Lizenz, sofern sich aus der Abbildungslegende nichts anderes ergibt. Sofern das betreffende Material nicht unter der genannten Creative Commons Lizenz steht und die betreffende Handlung nicht nach gesetzlichen Vorschriften erlaubt ist, ist für die oben aufgeführten Weiterverwendungen des Materials die Einwilligung des jeweiligen Rechteinhabers einzuholen.

Weitere Details zur Lizenz entnehmen Sie bitte der Lizenzinformation auf http://creativecommons.org/ licenses/by/4.0/deed.de.

\section{Literatur}

Ackermann, S. (2011). Klassengespräch im Mathematikunterricht. Eine Pilotstudie im Rahmen des Projekts „Persönlichkeits- und Lernentwicklung von Grundschulkindern“. Reihe Studium und Forschung, Bd. 19. Zentrum für Lehrerbildung der Universität Kassel

Biedermann, H., Weber, C., Herzog-Punzenberger, B., \& Nagel, A. (2015). Kapitel 4: Auf die Mitschüler/ innen kommt es an? Schulische Segregation - Effekte der Schul- und Klassenzusammensetzung in der Primarstufe und der Sekundarstufe I. In M. Bruneforth, F. Eder, K. Krainer, C. Schreiner, A. Seel \& C. Spiel (Hrsg.), Fokussierte Analysen bildungspolitischer Schwerpunktthemen. Nationaler Bildungsbericht Österreich 2015, Bd. 2. https://doi.org/10.17888/nbb2015-2-4.

Bos, W., Valtin, R., Hußmann, A., Wendt, H., \& Goy, M. (2017). IGLU 2016: Wichtige Ergebnisse im Überblick

Bürgermeister, A., \& Saalbach, H. (2018). Formatives Assessment: Ein Ansatz zur Förderung individueller Lernprozesse. Psychologie in Erziehung und Unterricht, 65, 194-205.

Bürgermeister, A., Große, G., Leuchter, M., Studhalter, U., \& Saalbach, H. (2018). Interaktion von pädagogischen Fachkräften und Kindern in naturwissenschaftlichen Lerngelegenheiten im Kindergarten: Ein Blick auf die Quantität kindlicher Redebeiträge. Frühe Bildung, 8(1), 13-21.

Decristan, J., Fauth, B., Heide, E., Locher, F., Troll, B., Kurucz, C., \& Kunter, M. (2020). Individuelle Beteiligung am Unterrichtsgespräch in Grundschulklassen: Wer ist (nicht) beteiligt und welche Konsequenzen hat das für den Lernerfolg? Zeitschrift für Pädagogische Psychologie. https://doi.org/10. 1024/1010-0652/a000251.

Decristan, J., Hondrich, A. L., Büttner, G., Hertel, S., Klieme, E., Kunter, M., Lühken, A., Adl-Amini, K., Djakovic, S., Mannel, S., Naumann, A., \& Hardy, I. (2015). Impact of additional guidance in science education on primary students' conceptual understanding. The Journal of Educational Research, 108, $358-370$.

Denn, A., Hess, M., \& Lipowsky, F. (2019). Hängen das Leistungsniveau und die Leistungsheterogenität von Grundschulklassen mit dem Anteil lehrerzentrierter Unterrichtsphasen im Deutsch- und Mathematikunterricht zusammen? Ergebnisse der PERLE-Studie. Zeitschrift für Grundschulforschung, $10(1), 162-176$.

Dubowy, M., Ebert, S., von Maurice, J., \& Weinert, S. (2008). Sprachlich-kognitive Kompetenzen beim Eintritt in den Kindergarten. Ein Vergleich von Kindern mit und ohne Migrationshintergrund. Zeitschrift für Entwicklungspsychologie und Pädagogische Psychologie, 40(3), 124-134.

Ewerhardy, A., Kleickmann, T., \& Möller, K. (2012). Fördert ein konstruktivistisch orientierter naturwissenschaftlicher Sachunterricht mit strukturierenden Anteilen das konzeptuelle Verständnis bei den Lernenden? Zeitschrift für Grundschulforschung, 5(1), 76-88.

Fthenakis, W.E. (2009). Ko-Konstruktion: Lernen durch Zusammenarbeit. Kinderzeit: Zeitschrift für Pädagogik und Bildung, 2009(3), 8-13.

Giest, H., Michalik, K., Franz, U., Haltenberger, M., Kantreiter, J., \& Hartinger, A. (2021). Editorial. In U. Franz, H. Giest, M. Haltenberger, A. Hartinger, J. Kantreiter \& K. Michalik (Hrsg.), Sache 
und Sprache. Probleme und Perspektiven des Sachunterrichts, (Bd. 31, S. 9-13). Bad Heilbrunn: Klinkhardt.

Haag, N., Heppt, B., Stanat, P., Kuhl, P., \& Pant, H. A. (2013). Second language learners' performance in mathematics: disentangling the effects of academic language features. Learning and Instruction, 28, 24-34.

Hardy, I., Sauer, S., \& Saalbach, H. (2019). Frühe Sprachliche Bildung im Kontext Naturwissenschaften: Effekte einer Intervention im Kindergarten. Psychologie in Erziehung und Unterricht, 3/2019, 196216.

Hartig, J., Jude, N., \& Wagner, W. (2008). Methodische Grundlagen der Messung und Vorhersage sprachlicher Kompetenzen. In E. Klieme (Hrsg.), Unterricht und Kompetenzerwerb in Deutsch und Englisch. Ergebnisse der DESI-Studie (S. 34-54). Weinheim: Beltz.

Hartinger, A., Grygier, P., Ziegler, F., Kullmann, H., \& Tretter, T. (2014). Individuelle Förderung beim naturwissenschaftlichen Lernen im Sachunterricht der Grundschule. Zeitschrift für Grundschulforschung, 7(2), 102-114.

Hayes, A., \& Krippendorff, K. (2007). Answering the call for a standard reliability measure for coding data. Communication Methods and Measures, 1, 77-89. https://doi.org/10.1080/19312450709336664.

Herwartz-Emden, L., \& Küffner, D. (2006). Schulerfolg und Akkulturationsleistungen von Grundschulkindern mit Migrationshintergrund. Zeitschrift für Erziehungswissenschaft, 9(2), 240-254.

Herzog-Punzenberger, B., \& Schnell, P. (2012). Kapitel 6: Die Situation mehrsprachiger Schüler/innen im österreichischen Schulsystem. In B. Herzog-Punzenberger (Hrsg.), Fokussierte Analysen bildungspolitischer Schwerpunktthemen. Nationaler Bildungsbericht Österreich 2012, Bd. 2. https://doi.org/10. 17888/nbb2012-2-6.

Inagaki, K., Hatano, G., \& Morita, E. (1998). Construction of mathematical knowledge through wholeclass discussion. Learning and Instruction, 8(6), 503-526.

Ing, M., Webb, N. M., Franke, M. L., Turrou, A. C., Wong, J., Shin, N., \& Fernandez, C. H. (2015). Student participation in elementary mathematics classrooms: the missing link between teacher practices and student achievement? Educational Studies in Mathematics, 90(3), 341-356.

Kähler, J., Hahn, I., \& Köller, O. (2021). Naturwissenschaftliche Kompetenz in der Grundschule: Effekte von Familienmerkmalen und Klassenkomposition. Zeitschrift für pädagogische Psychologie, 0, 1-15.

Kahlert, J. (2009). Der Sachunterricht und seine Didaktik (3. Aufl.). Bad Heilbrunn: Klinkhardt.

Kempert, S., Schalk, L., \& Saalbach, H. (2019). Sprache als Werkzeug des Lernens: Ein Überblick zu den kommunikativen und kognitiven Funktionen der Sprache und deren Bedeutung für den fachlichen Wissenserwerb. Psychologie in Erziehung und Unterricht, 66, 176-195.

Kleickmann, T., Vehmeyer, J., \& Möller, K. (2010). Zusammenhänge zwischen Lehrervorstellungen und kognitivem Strukturieren im Unterricht am Beispiel von Scaffolding-Maßnahmen. Unterrichtswissenschaft, 38(3), 210-228.

Lange, K., Ohle-Peters, A., Kleickmann, T., Kauertz, A., Möller, K., \& Fischer, H. (2015). Zur Bedeutung von Fachwissen und fachdidaktischem Wissen für Lernfortschritte von Grundschülerinnen und Grundschülern im naturwissenschaftlichen Sachunterricht. Zeitschrift für Grundschulforschung, 8, 23-38.

Leisen, J. (2015). Fachlernen und Sprachlernen! Bringt zusammen, was zusammen gehört! Zeitschrift des Deutschen Vereins zur Förderung des mathematischen und naturwissenschaftlichen Unterrichts e. V., 68(3), 132-137.

Leuchter, M., \& Saalbach, H. (2014). Verbale Unterstützungsmaßnahmen im Rahmen eines naturwissenschaftlichen Lernangebots in Kindergarten und Grundschule. Unterrichtswissenschaft, 42, 117-131.

Mannel, S., Meschede, N., \& Hardy, I. (2019). Effects of contingent support in primary science classroom discourse. In Tagungsbeitrag. EARLI Conference 2019. RWTH Aachen.

Martschinke, S., \& Kammermeyer, G. (2003). Jedes Kind ist anders. Jede Klasse ist anders. Ergebnisse aus dem KILIA-Projekt zur Heterogenität im Anfangsunterricht. Zeitschrift für Erziehungswissenschaft, 6(2), 257-275.

Mercer, N., \& Dawes, L. (2014). The study of talk between teachers and students, from the 1970s until the 2010s. Oxford Review of Education, 40(4), 430-445.

Möller, K. (2007). Naturwissenschaftlicher Sachunterricht. Kinder beim Erlernen von Naturwissenschaften helfen. Grundschulmagazin, 07(1), 8-10.

Muhonen, H., Rasku-Puttonen, H., Pakarinen, E., Poikkeus, A., \& Lerkkanen, M. (2016). Scaffolding through dialogic teaching in early school classrooms. Teaching and Teacher Education, 55, 143-154.

Muthén, L. K., \& Muthén, B.O. (2017). Mplus user's guide (8. Aufl.). Los Angeles: Muthén \& Muthén. (1998-2017) 
Pauli, C., \& Lipowsky, F. (2007). Mitmachen oder zuhören? Mündliche Schülerinnen- und Schülerbeteiligung im Mathematikunterricht. Unterrichtswissenschaft, 35(2), 101-124.

Quehl, T., \& Trapp, U. (2013). Sprachbildung im Sachunterricht der Grundschule. Mit dem ScaffoldingKonzept unterwegs zur Bildungssprache. Münster: Waxmann.

Reiss, K., Sälzer, C., Schiepe-Tiska, A., Klieme, E., \& Köller, O. (Hrsg.). (2016). PISA 2015. Eine Studie zwischen Kontinuität und Innovation. Münster, New York: Waxmann.

Saalbach, H., Gunzenhauser, C., Kempert, S., \& Karbach, J. (2016). Der Einfluss von Mehrsprachigkeit auf mathematische Fähigkeiten bei Grundschulkindern mit niedrigem sozioökonomischem Status. Frühe Bildung, 5(2), 73-81. https://doi.org/10.1026/2191-9186/a000255.

Sedova, K., Sedlacek, M., Svaricek, R., Majcik, M., Navratilova, J., Drexlerova, A., Kychler, J., \& Salamounova, Z. (2019). Do those who talk more learn more? The relationship between student classroom talk and student achievement. Learning and Instruction, 63, 101217.

Seidel, T., Rimmele, R., \& Prenzel, M. (2003). Gelegenheitsstrukturen beim Klassengespräch und ihre Bedeutung für die Lernmotivation. Videoanalysen in Kombination mit Schülerselbsteinschätzungen. Unterrichtswissenschaft, 31(2), 142-165.

Stanat, P. (2008). Heranwachsende mit Migrationshintergrund im deutschen Bildungswesen. In K. S. Cortina, J. Baumert, A. Leschinsky, K. U. Mayer \& L. Trommer (Hrsg.), Das Bildungswesen in der Bundesrepublik Deutschland: Strukturen und Entwicklungen im Überblick (S. 691-705). Hamburg: Rowohlt.

Studhalter, U., Leuchter, M., Tettenborn, A., Elmer, A., Edelsbrunner, P., \& Saalbach, H. (2021). Early science learning: the effects of teacher talk. Learning and Instruction, 71, 101371.

Tröbst, S., Kleickmann, T., Lange-Schubert, K., Rothkopf, A., \& Möller, K. (2016). Instructions and student's declining interest in science: an analysis of German fourth- and sixth-grade classrooms. American Educational Research Journal, 53(1), 162-193.

Van de Pol, J., Volman, M., Oort, F., \& Beishuizen, J. (2015). The effects of scaffolding in the classroom: support contingency and student independent working time in relation to student achievement, task effort and appreciation of support. Instructional Science, 43(5), 615-641.

Weiß, R. (2005). CFT 20-R. Grundintelligenztest Skala 2. Revision

Wood, D., Bruner, J. S., \& Ross, G. (1976). The role of tutoring in problem solving. Child Psychology \& Psychiatry \& Allied Disciplines, 17(2), 89-100. https://doi.org/10.1111/j.1469-7610.1976.tb00381. $\mathrm{x}$.

Wygotski, L. (1987). Arbeiten zur psychischen Entwicklung der Persönlichkeit. Ausgewählte Schriften, Bd. 2. Köln: Pahl-Rugenstein. 